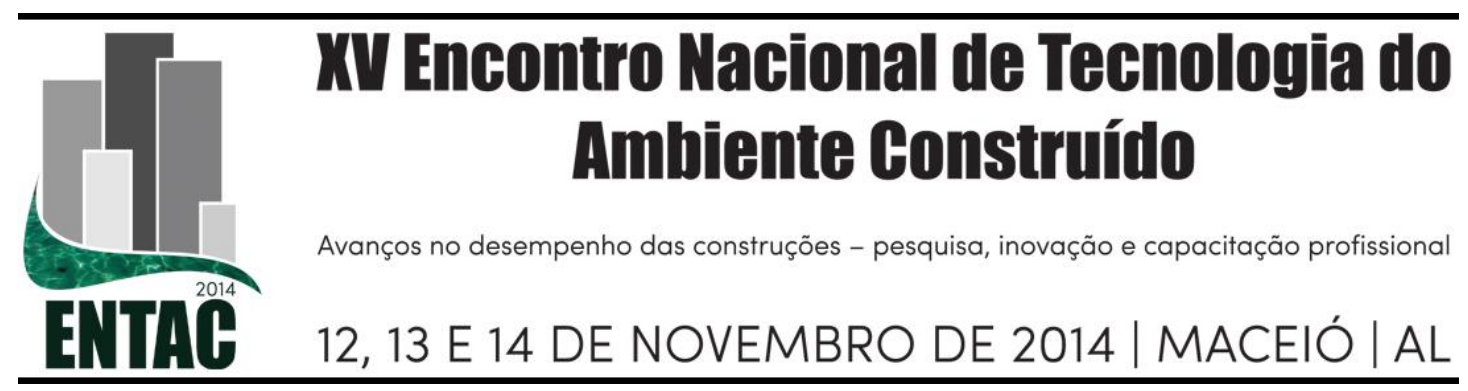

\title{
FELIZ E OS INDICATIVOS DE DESENVOLVIMENTO E SUSTENTABILIDADE PARA MUNICIPALIDADES
}

\author{
DE MOARES DE ZORZI, Lizia (1); GIULIANI KRÁS BORGES, Joice (2); \\ BASSO POLI, Cláudia Maria (3)
}

(1) Universidade Federal do Rio Grande do Sul, e-mail: liziadz@ gmail.com (2) Universidade Federal do Rio Grande do Sul, e-mail: joicekras@ hotmail.com, (3) Universidade Federal do Rio Grande do Sul, e-mail: claudiapoli@gmail.com

\begin{abstract}
RESUMO
Nas últimas décadas, foram criados diversos indicadores de aspectos ambientais, econômicos, sociais, éticos e culturais, que objetivaram fornecer uma contribuição ao diálogo da sustentabilidade. Para avaliar padrões de sustentabilidade e nortear rumos para o desenvolvimento de municípios, é importante a definição de indicadores. Neste contexto, têm-se destacado o município de Feliz, no interior do estado do Rio Grande do Sul. Localizado na região conhecida como Vale da Felicidade, o pequeno município foi classificado no ano de 2012 como o município de maior ISDM (Indicador Social de Desenvolvimento dos Municípios) do estado, e o quinto maior ISDM do país. Anteriormente a isso, o município já foi classificado, no ano de 1998, como o município brasileiro de maior IDH (Índice de Desenvolvimento Humano) e, no ano de 2010, com o menor índice de analfabetismo do país. Este estudo, desenvolvido no Núcleo Orientado de Inovação da Edificação (NORIE) da Universidade Federal do Rio Grande do Sul, tem por objetivo avaliar o desenvolvimento e a sustentabilidade desta cidade através da análise de indicadores. Com isso, a pesquisa visa contribuir para a aplicação de padrões de sustentabilidade para outras cidades. Para a realização do estudo, inicialmente foram analisadas as possíveis contribuições de alguns indicadores para as políticas publicas e avaliadas as dimensões do ISDM relacionadas à renda, habitação, educação, trabalho, segurança e saúde, englobando algumas das questões mais prementes nas políticas públicas. Os resultados apontaram quais as principais potencialidades e problemas do município de Feliz, fornecendo diretrizes para a proposição de políticas públicas dedicadas à melhoria das condições de vida e sustentabilidade do município.
\end{abstract}

Palavras-chave: Indicadores de Desenvolvimento, Sustentabilidade dos Municípios, Município de Feliz.

\begin{abstract}
In recent decades, several indicators of environmental, economic, social, ethical and cultural aspects, aimed to provide a contribution to the dialogue of sustainability were created. To evaluate sustainability standards and guide directions for the development of the cities, it is important to define indicators.In this context, have highlighted the city of Feliz, in the state of Rio Grande do Sul. Located in the area known as Happy Valley, the region was rated in 2012 as the largest city of ISDM (Social Indicator Development of the Countries) of the state, and the fifth largest ISDM of the country. Prior to this, the small town has been classified, in 1998, as the Brazilian municipality of highest HDI (Human Development Index) and, in 2010, as the lowest illiteracy rate in the country. This study was developed at the Center of the Building Oriented to Innovation (NORIE) of the Federal University of Rio Grande do Sul, aims to assess the development and sustainability of this city through the analysis of indicators. This research aims to contribute to the implementation of standards for the sustainability of other cities. The study initially analyzed the possible contributions of some indicators for public policy and evaluated the size of the Social Indicator Municipal Development (ISDM) related to income, housing, education, labor, health and safeyt, encompassing some of the most pressing issues in public policy. The results indicated that the
\end{abstract}


main potentialities and problems of the city of Feliz providing guidelines for proposing public policies aimed at improving living conditions and sustainability of the municipality.

Keywords: Development Indicators, Sustainable Cities, City of Feliz.

\section{INTRODUÇÃOO}

Diversos indicadores de aspectos ambientais, econômicos, sociais, éticos e culturais, que objetivaram fornecer uma contribuição ao diálogo da sustentabilidade, foram criados nas últimas décadas. Segundo Louette (2007), para definir padrões sustentáveis de desenvolvimento, tornou-se necessário definir indicadores que avaliem e monitorem esses padrões sustentáveis. Dentre eles destacam-se: a PE (Pegada Ecológica) e o HPI (Happy Planet Index), o IDH (Índice de Desenvolvimento Humano), e o ISDM (o Indicador Social de Desenvolvimento Municipal). Os dois primeiros costumam ser utilizados apenas na escala global, já os dois últimos estão sendo aplicados para contribuir com políticas públicas locais e regionais.

Neste contexto, têm-se destacado o município gaúcho de Feliz, objeto de estudo desta pesquisa. Localizado na região do Vale do Caí, também conhecido como Vale da Felicidade, o município com pouco menos de 13 mil habitantes foi classificado no ano de 2012 como o município de maior ISDM do estado, e o quinto maior ISDM do país (FGV, 2012). Anteriormente a isso, ele foi classificado, no ano de 1998, como o município brasileiro de maior IDH e, no ano de 2010, como o município com menor índice de analfabetismo do país (FELIZ, 2013).

Para a realização deste estudo de caso, desenvolvido no Núcleo Orientado de Inovação da Edificação (NORIE) da Universidade Federal do Rio Grande do Sul, foram analisadas as variáveis relacionadas a renda, habitação, educação, trabalho, segurança e saúde do ISDM. A partir destas análises, foram observadas as principais potencialidades e problemas locais e apresentadas diretrizes para a proposição de políticas públicas dedicadas à melhoria das condições de vida e sustentabilidade no município de Feliz, que deverá servir de modelo para outras pequenas municipalidades.

\section{FUNDAMENTAÇÃO}

Os indicadores de sustentabilidade surgiram com a Conferência Mundial sobre o Meio Ambiente, Rio-92, conforme a Agenda 21. A intenção era definir padrões sustentáveis de desenvolvimento que medissem aspectos ambientais, econômicos, sociais, éticos e culturais. Para isso, tornou-se necessário definir indicadores que mensurassem, monitorassem e avaliassem as realidades locais (GESINALDO; CÂNDIDO, 2012).

Para a realização deste estudo, inicialmente foram analisadas as possíveis contribuições dos seguintes indicativos para as políticas publicas: a PE (Pegada Ecológica), o HPI (Happy Planet Index), o IDH (Índice de Desenvolvimento Humano), e o ISDM (Indicador Social de Desenvolvimento dos Municípios). Posteriormente, o estudo foi aprofundado a partir dos dados do ISDM para o município de Feliz. A escolha por esse indicador deveu-se ao fato de ele abranger variáveis importantes para as políticas públicas locais. De acordo com Barbosa e colaboradores (2012) para compreender o desenvolvimento de um local devem ser considerados os aspectos locais relacionados à melhoria da qualidade de vida das pessoas e à conservação do meio ambiente. Estes fatores estão inter-relacionados e são interdependentes, já que o aspecto econômico implica em aumento da renda e riqueza, além de condições dignas de trabalho e saúde.

$\mathrm{O}$ indicador de PE refere-se à quantidade de área produtiva necessária para fornecer os recursos necessários às atividades humanas (GUIMARÃES; FEICHAS, 2009), sendo calculado a partir de dados sobre consumo da população e os recursos necessários à 
produção dos produtos e serviços consumidos. Ele indica, portanto, a sustentabilidade do estilo de vida de indivíduos, produtos e serviços, organizações, setores industriais, vizinhanças, cidades, regiões e nações. Os seus relatórios indicam que as populações tecnologicamente avançadas, em geral, têm PE maiores do que as subdesenvolvidas.

Lançado em 2006 pela New Economics Foundation (NEF), o HPI mede a eficiência com a qual os países convertem seus recursos naturais em bem-estar para os seus cidadãos (MARKS et al., 2009). Sendo assim, uma sociedade bem-sucedida é aquela na qual se pode levar uma boa vida sem custar a Terra. O cálculo do HPI incorpora três indicadores: a PE, a satisfação com a vida, e a expectativa de vida (LOUETTE, 2007). O HPI identifica a saúde e a experiência positiva de vida como objetivos humanos universais, e os recursos naturais como fundamentais aos sistemas humanos dependentes de insumos, devendo ser utilizado como um indicador a título de progresso (NEF, 2012). O relatório de 2012 mostrou que dezenas de países de alta renda são rebaixados consideravelmente no ranking do HPI por suas grandes PE, e apontou as áreas nas quais os países devem se concentrar as suas políticas (ABDALLAH et al., 2012).

Bastante utilizado para avaliar tanto realidades a nível global quanto local, IDH foi proposto em 1990 pelo Programa das Nações Unidas para o Desenvolvimento como ferramenta para mensurar o desenvolvimento econômico e humano, sintetizando quatro aspectos: expectativa de vida, taxa de alfabetização, escolaridade e PIB per capita (BRAGA et al., 2004). De acordo com os autores, o IDH visa captar em um único número uma realidade complexa sobre desenvolvimento humano e privações de necessidades básicas. Para aprofundar os estudos sobre as realidades a nível local, a Fundação Getúlio Vargas criou, em 2012, o ISDM (AMORIM; SANTOS; CÂNDIDO, 2008). O indicador abrange as principais dimensões mais prementes nas políticas públicas e torna possível a comparação do desempenho dos municípios entre si, além da comparação ao longo do tempo.

\section{MÉTODO}

Para a realização do estudo, foram avaliadas as dimensões relacionadas à renda, habitação, educação, trabalho, segurança e saúde, componentes do ISDM. A análise dos dados do indicador aponta quais são os principais problemas e potencialidade da cidade. Através de entrevistas e outros levantamentos realizados in loco pode-se entender o porquê dos resultados, e quais os aspectos que devem ser tratados como prioridade pelas políticas públicas.

Como algumas das variáveis que compõem as dimensões do ISDM apresentam diferentes unidades de medidas, sendo algumas positivas (quanto maior o indicador melhor será índice) e outras negativas (quanto maior o indicador pior será o índice), os seus valores foram transformados em índices de zero a um, facilitando a compreensão e comparação dos dados. Seguindo o método de Martins e Cândido (2008), para a transformação dos dados do ISDM em índices comparativos, foi realizada operacionalização a partir da Fórmula 1, utilizada quando a relação é positiva, e da Fórmula 2, quando a relação é negativa.

$$
\begin{gathered}
\mathrm{I}=(\mathrm{x}-\mathrm{m}) /(\mathrm{M}-\mathrm{m}) \\
\mathrm{I}=(\mathrm{M}-\mathrm{x}) /(\mathrm{M}-\mathrm{m})
\end{gathered}
$$

I = Índice calculado para cada Estado e municípios analisados;

$\mathrm{x} \quad=$ Valor de cada variável em cada Estado ou Município;

$\mathrm{m} \quad=$ Valor mínimo identificado nessas localidades; 
Foram considerados valores mínimos (m) e máximos (M) de cada variável, os valores extremos obtidos na base de dados do ISDM para os municípios do Rio Grande do Sul. A partir dos valores extremos e dos de Feliz (x) foram obtidos, então, os índices comparativos (I) que variam de zero a um. Para classificação e posterior elaboração de ranking, os valores dos índices foram divididos em quatro níveis, como em estudo de Martins e Cândido (2008), conforme pode ser observado do Quadro 1.

\section{Quadro 1 - Classificação dos índices}

\begin{tabular}{|c|c|c|}
\hline Índice $(\mathbf{0}-\mathbf{1})$ & Desempenho & Classificação \\
\hline $0,7501-1,000$ & Ideal & \\
\hline $0,5001-0,7500$ & Aceitável & \\
\hline $0,2501-0,5000$ & Alerta & \\
\hline $0,0000-0,2500$ & Crítica & \\
\hline
\end{tabular}

Fonte: Martins e Cândido (2008).

\section{RESULTADOS}

O relatório do ISDM de 2010 revela uma desigualdade regional em termos multidimensionais no Brasil. Há uma maior concentração de municípios acima da média nacional (cerca de $40 \%$ do total) na Região Sudeste. No estado do Rio Grande do Sul, $65 \%$ dos municípios apresentaram um desempenho acima da média nacional, sendo Feliz a cidade melhor classificada.

Feliz obteve um ISDM de 6,19 para o ano de 2010. Esse valor é bastante superior à média do estado $(5,55)$ e do país $(4,43)$, como pode ser observado no Quadro 2. Este quadro mostra também que a dimensão com maior valor para o Município foi a que trata da Saúde e Segurança $(8,97)$ e que a dimensão com o pior valor $(5,28)$ foi a da Habitação, possuindo essa um valor abaixo da média do estado $(5,40)$.

\section{Quadro 2 - O ISDM de Feliz, do Rio Grande do Sul e do Brasil}

\begin{tabular}{|c|c|c|c|c|c|c|}
\hline Local & ISDM & Habitação & Renda & Trabalho & Saúde e Segurança & Educação \\
\hline Feliz & 6,19 & 5,28 & 6,11 & 6,42 & 8,97 & 6,11 \\
\hline RS & 5,55 & 5,40 & 5,64 & 5,44 & 5,27 & 5,56 \\
\hline Brasil & 4,43 & 4,39 & 4,55 & 4,25 & 5,02 & 4,62 \\
\hline
\end{tabular}

Fonte: ISDM (2012)

Para melhor entender os resultados das diversas dimensões do ISDM foram, então, estudadas as sub-dimensões que compõem o indicador, apresentadas no Quadro 3.

\section{Quadro 3 - Dimensões que compõem o ISDM}

\begin{tabular}{|c|c|c|c|}
\hline Código & Dimensão & Sub-dimensão & Indicador \\
\hline H1 & \multirow{4}{*}{\multicolumn{2}{|c|}{ Habitação }} & Proporção de pessoas que vivem em domicílio atendido por coleta de lixo \\
\hline $\mathbf{H 2}$ & & & $\begin{array}{c}\text { Proporção de pessoas que vivem em domicílio com energia elétrica de } \\
\text { companhia distribuidora }\end{array}$ \\
\hline $\mathbf{H 3}$ & & & $\begin{array}{l}\text { Proporção de pessoas que vivem em domicílio com acesso à água } \\
\text { canalizada em pelo menos um cômodo }\end{array}$ \\
\hline H4 & & & $\begin{array}{c}\text { Proporção de pessoas que vivem em domicílio com esgotamento sanitário } \\
\text { do tipo rede geral de esgoto ou pluvial }\end{array}$ \\
\hline
\end{tabular}




\begin{tabular}{|c|c|c|c|}
\hline H5 & & & Proporção de pessoas que vivem em domicílio próprio de algum morador \\
\hline H6 & & & $\begin{array}{c}\text { Proporção de pessoas que vivem em domicílio que tem densidade de } \\
\text { moradores por dormitório inferior a } 2\end{array}$ \\
\hline $\mathbf{R 1}$ & \multirow{2}{*}{\multicolumn{2}{|c|}{ Renda }} & $\begin{array}{c}\text { Proporção de pessoas com renda domiciliar per capita abaixo da linha de } \\
\text { pobreza }\end{array}$ \\
\hline $\mathbf{R 2}$ & & & $\begin{array}{l}\text { Proporção de pessoas com renda domiciliar per capita abaixo da linha de } \\
\text { extrema pobreza }\end{array}$ \\
\hline T1_1 & \multirow{3}{*}{ Trabalho } & \multirow{2}{*}{ Geral } & Taxa de ocupação \\
\hline T1_2 & & & Taxa de formalização entre os empregados \\
\hline T2_1 & & Infantil & Taxa de trabalho infantil \\
\hline S1_1 & \multirow{6}{*}{ Saúde } & \multirow{3}{*}{ Infantil } & Taxa de mortalidade infantil, por mil nascidos vivos \\
\hline S1_2 & & & $\begin{array}{l}\text { Mortalidade proporcional por doenças com causas evitáveis de menores de } \\
5 \text { anos }\end{array}$ \\
\hline S1_3 & & & Proporção de nascidos vivos com baixo peso ao nascer \\
\hline S2_1 & & \multirow[b]{2}{*}{ Geral } & Proporção das adolescentes (10 a 19 anos) que já tiveram filho \\
\hline S2_2 & & & $\begin{array}{l}\text { Mortalidade proporcional por doenças com causas evitáveis na população } \\
\text { de } 5 \text { a } 74 \text { anos }\end{array}$ \\
\hline S3_1 & & Segurança & Taxa de homicídio, por cem mil habitantes \\
\hline E1_1 & \multirow{11}{*}{ Educação } & \multirow{2}{*}{ Ensino Infantil } & Proporção de crianças de 0 a 3 anos que frequentam creche \\
\hline E1_2 & & & Proporção de crianças de 4 a 6 anos que frequentam pré-escola \\
\hline E2_1 & & \multirow{6}{*}{$\begin{array}{c}\text { Ensino } \\
\text { Fundamental }\end{array}$} & Proporção de crianças de 8 ou 9 anos não alfabetizadas \\
\hline E2_2 & & & Proporção de adolescentes de 10 a 14 anos não alfabetizados \\
\hline E2_3 & & & Proporção de crianças de 7 a 14 anos que frequentam escola \\
\hline E2_4 & & & Proporção de crianças de 7 a 14 anos na série adequada para sua idade \\
\hline E2_5 & & & $\begin{array}{l}\text { Índice transformado na escala Ideb de proficiência Agregado para a } 4^{\mathrm{a}} \\
\text { série do EF ( } 5^{\circ} \text { ano EF) }\end{array}$ \\
\hline E2_6 & & & $\begin{array}{c}\text { Índice transformado na escala Ideb de proficiência Agregado } 8^{\text {a }} \text { série do } \\
\text { Ensino Fundamental }\left(9^{\circ} \text { ano EF) }\right.\end{array}$ \\
\hline $\mathbf{E 3 \_ 1}$ & & \multirow{3}{*}{$\begin{array}{l}\text { Ensino Médio e } \\
\text { Geral }\end{array}$} & Proporção de crianças de 15 a 17 anos que frequentam escola \\
\hline E3_2 & & & Proporção de jovens de 15 a 17 anos não alfabetizados \\
\hline E3_3 & & & Proporção de indivíduos com mais de 18 anos não alfabetizados \\
\hline
\end{tabular}

Fonte: ISDM (2012)

Para cada indicador, foi calculado um índice de desempenho que permitiu, além de uma melhor compreensão e comparação dos dados, a classificação do desempenho e o ranqueamento de prioridades para intervenção, conforme o Quadro 4 e a Figura 1.

\section{Quadro 4 - Índices do município de Feliz}

\begin{tabular}{|c|c|c|c|c|c|}
\hline \multicolumn{2}{|c|}{ Dimensão } & Índices & Desempenho & Classificação & Prioridade \\
\hline \multirow{6}{*}{ Habitação } & H1 & 0,9943 & Ideal & & $20^{\circ}$ \\
\hline & $\mathrm{H} 2$ & 0,9508 & Ideal & & $16^{\circ}$ \\
\hline & $\mathrm{H} 3$ & 0,9954 & Ideal & & $21^{\circ}$ \\
\hline & $\mathrm{H} 4$ & 0,1576 & Crítico & & $1^{\circ}$ \\
\hline & H5 & 0,5970 & Aceitável & & $4^{\circ}$ \\
\hline & H6 & 0,7901 & Ideal & & $11^{\circ}$ \\
\hline \multirow{2}{*}{ Renda } & R1 & 0,9880 & Ideal & & $19^{\circ}$ \\
\hline & $\mathrm{R} 2$ & 0,9965 & Ideal & & $21^{\circ}$ \\
\hline \multirow{3}{*}{ Trabalho } & T1_1 & 0,8508 & Ideal & & $13^{\circ}$ \\
\hline & T1_2 & 0,8956 & Ideal & & $14^{\circ}$ \\
\hline & T2_1 & 0,9744 & Ideal & & $17^{\circ}$ \\
\hline \multirow{6}{*}{$\begin{array}{c}\text { Saúde e } \\
\text { Segurança }\end{array}$} & $\begin{array}{l}\text { S1_1 } \\
\end{array}$ & 0,9776 & Ideal & & $18^{\circ}$ \\
\hline & $\overline{\text { S1_2 }}$ & 1 & Ideal & & $23^{\circ}$ \\
\hline & S1_3 & 0,7015 & Aceitável & & $8^{\circ}$ \\
\hline & S2_1 & 0,7554 & Ideal & & $9^{\circ}$ \\
\hline & S2_2 & 0,2833 & Alerta & & $2^{\circ}$ \\
\hline & S3_1 & 1 & Ideal & & $24^{\circ}$ \\
\hline \multirow{2}{*}{ Educação } & E1_1 & 0,6283 & Aceitável & & $5^{\circ}$ \\
\hline & $\overline{\text { E1_2 }}$ & 0,7742 & Ideal & & $10^{\circ}$ \\
\hline
\end{tabular}




\begin{tabular}{|l|c|c|c|c|c|}
\hline \multirow{7}{*}{} & E2_1 & 1 & Ideal & & $25^{\circ}$ \\
\hline & E2_2 & 1 & Ideal & & $26^{\circ}$ \\
\hline & E2_3 & 0,9200 & Ideal & & $15^{\circ}$ \\
\hline & E2_4 & 0,6487 & Aceitável & & $6^{\circ}$ \\
\hline & E2_5 & 0,6580 & Aceitável & & $7^{\circ}$ \\
\hline & E2_6 & 0,8165 & Ideal & & $12^{\circ}$ \\
\hline & E3_1 & 0,5348 & Aceitável & & $3^{\circ}$ \\
\hline & E3_2 & 1 & Ideal & & $27^{\circ}$ \\
\hline & E3_3 & 1 & Ideal & & $28^{\circ}$ \\
\hline
\end{tabular}

Figura 1 - Gráfico com os índices para as diversas dimensões do ISDM

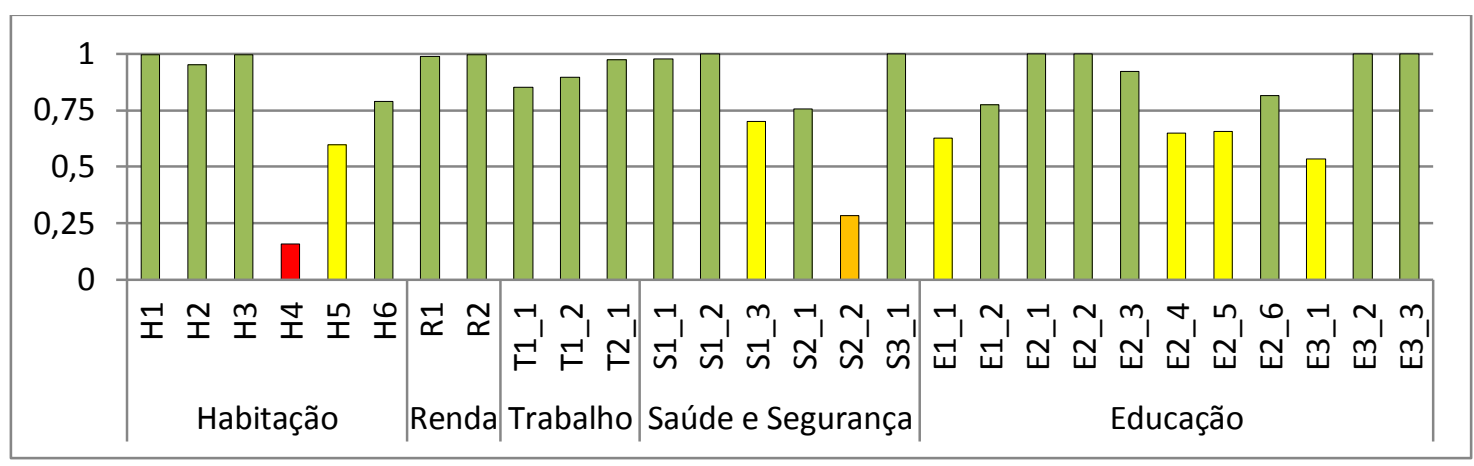

Os dados apresentados indicam o desempenho de cada sub-dimensão e quais devem ser as prioridades das políticas públicas. Na sequência, foi realizada a análise de cada dimensão considerando os principais índices observados, os dados disponibilizados pelo IBGE Cidades (2013) e as informações levantadas in loco.

\subsection{Habitação}

Conforme apresentado, a dimensão da Habitação foi a que apresentou o pior desempenho na cidade de Feliz devendo, portanto, ser prioridade das políticas públicas. O Quadro 4 mostra que a sub-dimensão que trata dos sistemas de esgoto das residências (H4), foi o único com desempenho considerado crítico.

De acordo com os dados do IBGE Cidades (2013), existe uma grande parcela da população vivendo em condições sanitárias inadequadas, muitas ainda utilizam valas, sem fossa séptica e sumidouro. A engenheira Buchweitz (2013) confirma que atualmente os sistemas de esgoto utilizados são predominantemente de fossa e filtro ou sumidouro. Na zona rural, aonde vive $24 \%$ da população, utiliza-se o sistema de sumidouro e valas de infiltração, sendo estes de responsabilidade dos proprietários. $\mathrm{Na}$ zona urbana, o sistema de fossa e filtro é o mais utilizado, e a rede canalizada leva o esgoto para o Rio Caí. O lançamento de esgotos in natura à rede pluvial contamina os corpos hídricos e possibilita a ocorrência de doenças e enfermidades de veiculação hídrica ameaçando a saúde da população. De acordo com a engenheira, não existe nenhuma companhia atendendo nem a rede pluvial nem a cloacal de esgoto do município. Entretanto, a prefeitura está em negociações com a Companhia Riograndense de Saneamento (CORSAN), para passar, mediante contrato de concessão, a responsabilidade destes serviços à mesma.

A outra sub-dimensão que não apresentou desempenho ideal, é a que trata do percentual de pessoal que vive em domicílio próprio (H5). Acredita-se que isso seja consequência do baixo rendimento nominal per capita e da distribuição desigual da renda. 


\subsection{Renda}

Apesar de a dimensão da renda ter um desempenho considerado como ideal, alguns problemas foram identificados. A cidade ainda deve buscar soluções para reduzir a pobreza (R1) cuja incidência é de $15,95 \%$. O rendimento nominal mediano per capita no município é de R \$ 655,00, na Zona Rural e R\$ 765,00 na Zona Urbana. Analisando os dados do IBGE Cidades (2013) sobre os rendimentos nominais mensais da população de Feliz, percebe-se que um elevado percentual da população (aproximadamente 43\%) ganha menos do que um salário mínimo. Estes valores indicam uma má distribuição de renda.

O Índice de Gini mede o grau de concentração de renda em um determinado grupo com valores entre zero, correspondente à completa igualdade de renda, e um, correspondente a total desigualdade. O valor para Feliz é de 0,4, estando bastante inferior a média do estado $(0,59)$ e a do país $(0,4)$. Se compararmos o índice com o do município de Trabiju/SP, considerada a cidade com melhor ISDM, ou com o de São Vedelino/RS, classificada em primeiro lugar na dimensão renda do ISDM, ambas as cidades com 0,35 de valor no Índice de Gini percebemos, novamente, que a renda de Feliz deveria ser mais bem distribuída para que houvesse um melhor desempenho geral.

A respeito da pobreza subjetiva, que se apoia no sentimento de impotência e exclusão social, Feliz tem um percentual de $10,79 \%$, bastante inferior ao da pobreza financeira real da cidade, que é de $15,95 \%$. Essa diferença mostra que apesar da baixa renda e da sua distribuição desigual, ainda há um grande percentual da população pobre que não se sente impotente e excluído da sociedade. Isso se deve ao fato de o percentual da população em extrema pobreza (R2) ser zero, e às características específicas da cidade como, por exemplo, a presença de hortas nas residências.

\subsection{Trabalho}

O desempenho de Feliz nesta dimensão foi considerado ideal. Como se pode observar no Quadro 4, a taxa de desempregados na cidade (T1_1) é pequena, e a de trabalho infantil (T2_1) é ainda menor. Acredita-se que a dimensão do trabalho tenha um valor alto em consequência de outra dimensão, a educação que, de acordo com Bublitz (2011), é o principal alvo das políticas públicas. Grande parte da população adulta consegue emprego dentro e fora da cidade, pois teve acesso à educação e as crianças não trabalham, pois estudam.

\subsection{Saúde e Segurança}

A dimensão que trata da saúde e da segurança foi a que apresentou o melhor desempenho no ISDM da cidade de Feliz, apesar de algumas das suas variáveis (S1_3 e S2_2) apresentarem desempenhos ruins, como mostrou o Quadro 4. Considerada como ideal, a taxa de mortalidade infantil (S1_1) no município de Feliz é 7,46, estando bastante abaixo tanto da média do estado $(11,17)$ quanto da média das $10 \%$ melhores classificadas $(11,21)$.

A mortalidade proporcional por doenças evitáveis em crianças com menos de cinco anos (S1_2) também é ideal, com valor igual a zero, assim como o percentual de adolescentes até 19 anos que já tiveram filhos (S2_1). Acredita-se que isso seja consequência da boa escolarização da população e, principalmente do programa de saúde da família.

O índice de nascidos vivos com baixo peso ao nascer (S1_3), não apresenta um desempenho ideal. Isto é consequência, principalmente, da má distribuição de renda e dos baixos salários de quase metade da população. $O$ outro índice que não apresenta desempenho satisfatório nesta dimensão é o que trata do percentual de mortalidade 
proporcional por doenças com causas evitáveis na população de 5 a 74 anos (S2_2). Este índice foi classificado como alerta tendo o segundo pior desempenho entre as subdimensões consideradas no estudo, apesar de o seu percentual $(71,67)$ ser bastante abaixo da média do estado $(77,17)$ e das cidades mais bem classificadas $(74,95)$.

Para reduzir o percentual de mortes por causas evitáveis, seria importante aumentar o atendimento do Sistema Único de Saúde (SUS), que conta com apenas quatro unidades. Além disso, analisando os dados do IBGE Cidades (2013) sobre as principais causas de morte, também se percebe que muitas mortes poderiam ser evitadas se houvesse um nível maior de especialização médica na cidade. Se a medicina na cidade fosse mais desenvolvida o percentual de mortes cujas causas são desconhecidas (28\%) provavelmente seria menor.

Sobre a segurança, o ISDM considera apenas a taxa de homicídios por cem mil habitantes (S3_1). Na cidade de Feliz este valor é zero, sendo o índice da dimensão considerado ideal, desta forma. Acredita-se que a cidade seja bastante segura em função da boa educação da população e, também, da baixa taxa de desemprego.

\subsection{Educação}

O ISDM da cidade, na dimensão da educação $(6,11)$, é bastante acima da média do estado do Rio Grande do Sul $(5,56)$ e do país $(4,62)$, conforme o quadro 2.

De acordo com o senso de 2010 do IBGE, a cidade de Feliz é a primeira no ranking da alfabetização brasileira (E2_1 e E2_2), e possui apenas 97 pessoas com mais de 15 anos não alfabetizadas (E3_2 e E3_3). Estes dados fazem com que o município apresente um índice de satisfação na educação de $80 \%$ por parte dos moradores, segundo pesquisa realizada pela Prefeitura em 2013. Acredita-se que este resultado seja influenciado por fatores históricos e culturais como, por exemplo, o pequeno porte da municipalidade, que aproxima o poder público da comunidade, e a colonização europeia, que tradicionalmente valoriza a educação.

O prefeito Cesar Luiz Assmann afirma que a educação é prioridade nos investimentos. Os recursos na área ultrapassam $25 \%$ do orçamento. Os professores são valorizados, e uma politica de qualificação é estimulada. Os programas de esporte, lazer e ensino garantem atividades que envolvem mais 2.300 pessoas. Oficinas de futebol, entre outras e cursos de idiomas são oferecidos gratuitamente a toda a população (BUBLITZ, 2011). Apesar disso, como pode ser observado no Quadro 9, algumas variáveis que compõem a dimensão educação não apresentam um desempenho ideal. A Proporção de crianças com até três anos que frequenta creche (E1_1) é extremamente baixa, pois a prefeitura não está conseguindo suprir a demanda local.

\section{CONSIDERAÇÕES FINAIS}

Conforme mostrou a pesquisa, indicadores são ferramentas importantes para a promoção do desenvolvimento e da sustentabilidade nas nações, estados e municípios. Neste contexto, o ISDM é conveniente por observar diferentes áreas do desenvolvimento e, ao mesmo tempo, fornecer uma medida sintética de bem-estar. Contribui para a formulação de políticas públicas e para melhor informar a sociedade sobre as condições de vida em um determinado local. Quanto mais heterogêneas forem as diferenças de um local, mais importante é a abordagem de múltiplos fatores.

O estudo de caso mostrou que, apesar do bom desempenho nos indicadores de educação, IDH e ISDM, o município de Feliz ainda precisa melhorar em muitos aspectos. A principal dimensão do ISDM que precisa ser melhorada para aumentar a sustentabilidade e o desenvolvimento local é a da habitação. A sua melhoria está 
diretamente relacionada à necessidade de implantação de um sistema de esgotamento sanitário. Como a prefeitura já está em negociação com a CORSAN para isso, acreditase que em breve o desempenho desta dimensão melhore. Também deverá contribuir para esta melhora o Plano Municipal de Saneamento Básico, recentemente criado pela Prefeitura, que prevê a criação de projetos de educação ambiental para conscientizar a população a respeito da importância da implantação de um sistema de esgotamento sanitário e necessidade da participação popular para o sucesso do mesmo.

Com relação à renda, constatou-se que o problema está, principalmente, na sua má distribuição, o que faz com que um percentual muito grande da população receba menos do que o salário mínimo. Acredita-se que, para que este cenário melhore, deva-se investir na qualificação profissional e incentivar a criação de empresas de pequeno porte. Para estimular a criação de empregos, seria interessante o desenvolvimento de uma Política Tarifária de Incentivo Fiscal.

A melhora no trabalho e na renda também resultaria em melhora a saúde reduzindo, por exemplo, a taxa de baixo peso ao nascer das crianças. Verificou-se que a cidade precisa ampliar o número de unidades de atendimento do Sistema Único de Saúde e buscar a qualificação do serviço através de uma maior especialização da medicina. Com isso, muitas mortes poderiam ter as suas causas esclarecidas e até serem evitadas.

Apesar de a educação ser prioridade nos investimentos da prefeitura e de ela já ter obtido o título de município mais alfabetizado no Brasil, observa-se que ela ainda apresenta carências e que precisa melhorar em todos os níveis. È importante lembrar, ainda, que as melhoras na educação acarretam em melhoras nas demais dimensões.

\section{REFERÊNCIAS}

ABDAllaH, S.; MICHAELSON, J.; SHAH, S.; STOOL, L.; MARKS, N. Happy Planet Index: 2012. 2012. Report. Disponível em: <http://www.neweconomics.org/publications/entry/happy-planet-index-2012-report.>. Acesso em: 02 jul. 2013.

AMORIM, B.P.; SANTOS, J.A.; CÂNDIDO, G.A. Índice de sustentabilidade municipal e as suas relações com as políticas e ações para geração do desenvolvimento sustentável: um estudo aplicado na cidade de João Pessoa - PB. SEGeT - Simpósio de Excelência em Gestão e Tecnologia. Anal. 2008. Disponível em: < http://www.aedb.br/seget/artigos08/553_Artigo\%20SEGET.pdf>. Acesso em: Fev. 2014.

BARBOSA, R.F.; VERÍSSIMO, P.; SOUSA, J.E.; MELO, J.W.L.; PEREIRA, D.A.M. Análise da tríade da sustentabilidade na cidade de Guarabira -PB por meio do IDSM (Índice de Desenvolvimento Sustentável para Municípios). Anal. XXXII Encontro Nacional de Engenharia de Produção. Bento Gonçalvez. 2012.

BRAGA, T.M.; FREITAS, A.P.G.; DUARTE, G.S.; CAREPA-SOUZA, J. Índices de sustentabilidade municipal: o desafio de mensurar. Nova Economia: Belo Horizonte. n.14, p.11-33, Set/Dez. 2004.

BUBLITZ, J. Feliz é o município mais alfabetizado do Brasil. Site da Prefeitura de Feliz. 2011. Disponível em: <http://www.feliz.rs.gov.br/noticias/2011/06/22/en/feliz-e-o-municipiomais-alfabetizado-do-brasil/>. Acesso em: 12 jul. 2013.

BUCHWEITZ, A. As condições hidrossanitárias da cidade de Feliz/RS. Feliz, 10 jul. 2013. Entrevista concedida a Joice Krás e Cláudia Poli.

FELIZ. Prefeitura Municipal de Feliz. Disponível em: <http://www.feliz.rs.gov.br.>. Acesso em: 24 jun. 2013.

FUNDAÇÃO GETÚLIO VARGAS. Indicador Social de Desenvolvimento dos Municípios ISDM. Sumário Executivo. São Paulo, 2012. 
GESINALDO, R.F.B.; CÂNDIDO, G.A. Análise do índice de Desenvolvimento Sustentável para Municípios IDSM: O Caso de Guarabira - PB. Revista da Ciência da Administração Versão Eletrônica, v. 5, Jan./Jul. 2012.

GUIMARÃES, R.P.; FEICHAS, S.A.Q. Desafios na construção de indicadores de sustentabilidade. Campinas: Ambiente e Sociedade, v. XII, n. 2, p. 307-323, Jul/Dez. 2009.

IBGE Cidades. Dados sobre o município de Feliz/RS. 2013. Disponível em: <http://cidades.ibge.gov.br/xtras/perfil.php?lang=\&codmun=430810.> Acesso em: 24 abr. 2014.

LOUETTE, A. Indicadores de nações: uma contribuição ao diálogo da sustentabilidade: Gestão do conhecimento. 1.ed. São Paulo: WHH - Willis Harman House, 2007.

MARKS, N.; ABDALLAH, S.; MICHAELSON, J.; THOMPSON, S.; STEUER, N. The Happy Planet Index 2.0. 2009. Disponível em: $<$ http://www.neweconomics.org/publications/entry/the-happy-planet-index-2.>. Acesso em: 02 jul. 2013.

MARTINS, M. F.; CÂNDIDO, G. A. Metodologia para Construção e Análise de Índices de Desenvolvimento Sustentável: uma aplicação no Estado da Paraíba. João Pessoa - PB: Edições SEBRAE, 2008.

THE NEW ECONOMICS FOUNDATION. The Happy Planet Index: 2012 Report - a global index of sustainable well-being. 2012.

UNCED. United Nations Conference on Environment and Development: Agenda 21. New York: United Nations. 2012. Disponível em: $<\mathrm{http} / / /$ sustainabledevelopment.un.org/content/documents/Agenda21.pdf $>$. Acesso em: $04 \mathrm{abr}$. 2013. 